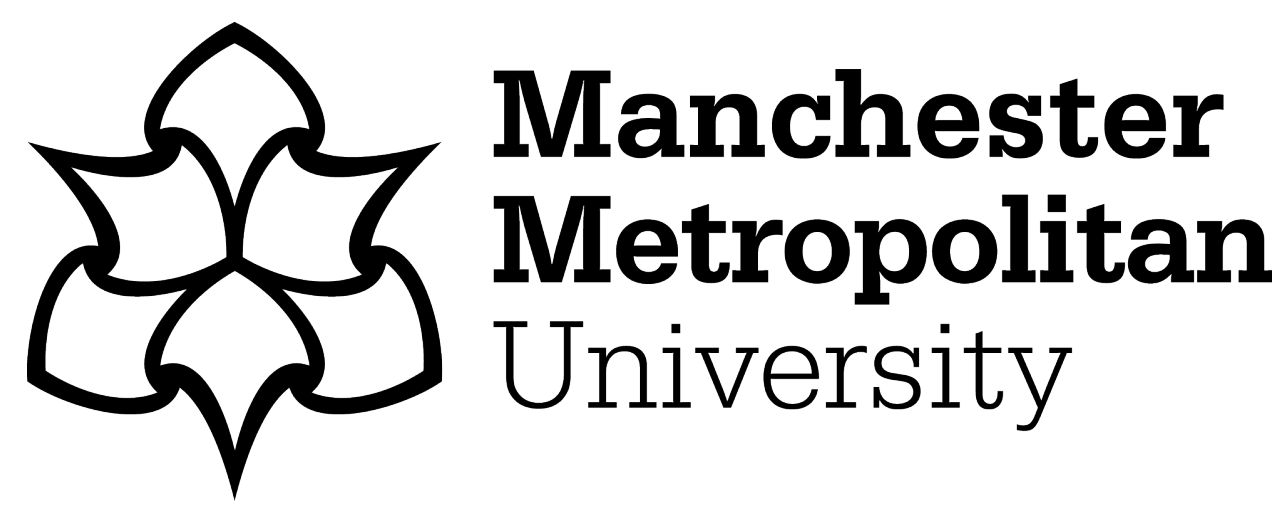

Betlem, Kai, Down, M, Foster, C, Akthar, S, Eersels, K, van Grinsven, B, Cleij, T, Banks, C and Peeters, Marloes (2018) Development of a Flexible MIPBased Biosensor Platform for the Thermal Detection of Neurotransmitters. MRS Advances, 3 (28). pp. 1569-1574. ISSN 2059-8521

Downloaded from: https://e-space.mmu.ac.uk/619904/

Version: Accepted Version

Publisher: Cambridge University Press

DOI: https://doi.org/10.1557/adv.2017.634

Please cite the published version 


\section{Development of a Flexible MIP-Based Biosensor Platform for the Thermal Detection of Neurotransmitters}

Kai Betlem ${ }^{1}$, Michael P. Down ${ }^{1}$, Christopher W. Foster ${ }^{1}$, Shamima Akthar ${ }^{1}$, K. Eersels ${ }^{2}$, B. van Grinsven ${ }^{2}$, T.J. Cleij ${ }^{2}$, C.E. Banks ${ }^{1}$, M. Peeters ${ }^{1 *}$

1) Manchester Metropolitan University, Faculty of Science and Engineering, Div. of Chemistry \& Environmental Science, John Dalton Building, Chester Street, M15GD, Manchester, United Kingdom. 2) Maastricht University, Maastricht Science Programme, P.O. Box 616, 6200 MD Maastricht, The Netherlands.

ABSTRACT

We have developed high affinity Molecularly Imprinted Polymers (MIPs) for neurotransmitters such as dopamine, noradrenaline and caffeine. These polymer particles are mixed within the bulk of screen-printed ink allowing masss-producible bulk modified MIP Screen-Printed Electrodes (MIP-SPES) to be realised. We have explored different SPE supporting surfaces, such as polyester, tracing paper and household-printing paper. The performance of those MIPSPEs is studied using the Heat-Transfer Method (HTM), a patented thermal method. With the combination of screen-printing techniques and thermal detection, it is possible to develop a portable sensor platform that is capable of low-cost and straightforward detection of biomolecules on-site. In the future, this unique sensor architecture holds great promise for the use in biomedical devices.

\section{INTRODUCTION}

Molecularly Imprinted Polymers (MIPs) are synthetic antibody mimics; similar to antibodies, they have high affinity for a chosen template molecule but their advantages include low-cost, superior chemical and thermal stability, and straightforward production process $[1,2]$. These polymeric receptors are widely used in purification and separation processes but have limited applications in the field of biosensors [3]. Key challenges in that area include difficulty to incorporate MIPs into suitable sensor platforms and the lack of low-cost and straightforward sensing strategies.

In this contribution, we will focus on using SPEs as sensor platforms since they are highly reproducible, can be used as disposable electrodes and their production process can be tailored with our in-house facilities. We were the first to report on novel and simple functionalization strategy for the direct incorporation of MIPs into SPEs [4]. These MIPSPEs will be combined with the Heat-Transfer Method (HTM) as sensing strategy. HTM is a patented thermal analysis technique [5] that relies on evaluating the thermal resistance at the solid-liquid interface. This method has commercial potential but is not currently 
suitable for mass-production due to various reasons, including the use of home-made design of the thermocouple device and flow cells.

We will address those issues by mass-producing MIP-SPEs for polymer particles designed for a variety of neurotransmitters and use of 3D-printing to develop and optimize flow cell design. This will overcome important hurdles that need to be taken for the commercial application of MIP-based thermal sensors, which holds great promise for future use in various areas such as biomedical devices or on-sight environmental analysis.

\section{EXPERIMENTAL}

\section{MIP synthesis}

MIPs for noradrenaline were produced as described in ref [4]. MIPs for caffeine were synthesized according to a protocol adapted from ref [6], using methacrylic acid and acrylamide as monomers in varying ratios.

For the selective recognition of dopamine, a range of polymers was produced to optimize specificity. Table 1 provides an overview of the chemicals and quantities that were used. Prior to the polymerisation, stabilisers in the monomer and crosslinker monomers were removed by filtering the solution over basic alumina oxide. Dopamine hydrochloride $(1 \mathrm{mmol})$ was then dissolved with the functional monomers $(2 \mathrm{mmol})$, ethylene gylcoldimethacrylate $(10 \mathrm{mmol})$ into a suitable porogen $(5 \mathrm{ml})$. Subsequently, the initiator azobisisobutyronitrile (50 mg AIBN) was added and the solution was purged with a gentle flow of nitrogen for $\sim 5 \mathrm{~min}$. The mixture was then polymerised under reflux at $60^{\circ} \mathrm{C}$ for $12 \mathrm{~h}$ and was ground to obtain microparticles. Extraction was performed with various solvents, including a methanol/water mixture $(12 \mathrm{~h})$, acetic acid combined with acetonitrile (48h) and finally methanol/water (12h). The extraction process was monitored by determining the amount of template in the filtrate. After extraction was complete, the polymers were ground to obtain microparticles and dried under vacuum for $12 \mathrm{~h}$. Particles were sieved to only obtain those with sizes smaller than $<10 \mu \mathrm{m}$. Non-Imprinted Polymers (NIPs) were prepared in the same manner but without addition of the template. Binding to the MIP and NIP was determined by optical batch rebinding experiments, demonstrating equilibrium was reached after a binding time of $1 \mathrm{~h}$. 


\begin{tabular}{|c|c|c|}
\hline MIP 1 & $\begin{array}{c}\text { Functional monomer (mmol) } \\
\text { 2-Hydroxyethyl methacrylate } \\
(\text { HEMA, } 2 \text { mmol })\end{array}$ & $\begin{array}{c}\text { Methanol / water } \\
(80 \%, 20 \%)\end{array}$ \\
\hline MIP 2 & $\begin{array}{c}\text { Itaconic acid } \\
(2 \mathrm{mmol})\end{array}$ & $\begin{array}{c}\text { Methanol } / \text { water } \\
(80 \%, 20 \%)\end{array}$ \\
\hline MIP 3 & $\begin{array}{c}\text { HEMA / Itaconic acid } \\
(1 \mathrm{mmol}, 1 \mathrm{mmol})\end{array}$ & $\begin{array}{c}\text { Methanol / water } \\
(80 \%, 20 \%)\end{array}$ \\
\hline MIP 4 & $\begin{array}{c}\text { HEMA } \\
(2 \mathrm{mmol})\end{array}$ & DMSO \\
\hline MIP 5 & $\begin{array}{c}\text { Itaconic acid } \\
(2 \text { mmol })\end{array}$ & DMSO \\
\hline MIP 6 & $\begin{array}{c}\text { HEMA / itaconic acid } \\
(1 \mathrm{mmol}, 1 \mathrm{mmol})\end{array}$ & DMSO \\
\hline
\end{tabular}

\section{Incorporating MIP particles into SPEs}

Details about the functionalization procedure are described in ref [4]. A carbon-graphite ink formulation (C2000802P2 from Gwent Electronic Materials Ltd) was printed onto substrates with different substrates, including polyester and paper, and cured at $60^{\circ} \mathrm{C}$ for $30 \mathrm{~min}$. The MIPs were incorporated into the bulk of the graphite ink on the basis of the weight percentage $M_{p}$ and $M_{i}$, where $M_{p}$ is the mass of the particulate, in this case the MIPs, and $M_{i}$ is the mass of the ink formulation used in the printing process, i.e. $=\left(M_{p} / M\right) \times 100.30 \%$ was chosen in this work because this is the balance between optimum MIP availability and conductivity of the ink. The particles are insulating and concentrations higher than $30 \%$ are not sufficiently conductive enough to be used in the screen-printing process. It was noted that the mixing of the MIPs with the graphite ink is strongly dependent on the hydrophobicity of the material.

\section{Thermal measurements}

MIP-SPEs were mounted into a home-made thermocouple device, which design is described in ref [5]. This was equipped with a automated NE500 syringe pump from ProSense (the Netherlands), allowing to control the inflow and flow rate of samples added to the flow cell. The flow cell was fabricated by $3 \mathrm{D}$-printing and a technical drawing (Solidworks 2016 3D CAD (SP4) Software package) is provided in Figure 1.

The model was printer utilising a FORM 2 stereolithography (SLA) 3DPrinter from FormLabs (USA) with a layer height of $25 \mu \mathrm{m}$. The model was printed utilising FORM 2 Clear Resin (GPCL04), before being bathed and washed in isopropanol to remove any uncured polymer resin. The flow cell channels were cleared using a syringe of isopropanol to ensure no polymer resin would block or vary the diameter of the flow cell channels during the final cure. The printed flow cell was then fully cured in a UV post-print chamber to ensure the complete polymerization of the material and the stabilization of the mechanical properties. 
A copper block is used to close off the flow cell and to serve as a heat sink. The temperature gradient is then determined as the difference between the temperature at which the copper is controlled $\left(\mathrm{T}_{1}\right)$ versus the temperature that is monitored in the liquid $\left(T_{2}\right)$ by a thermocouple type $\mathrm{K}$. In the design that we used the thermocouple is inserted at $1.7 \mathrm{~mm}$ above the electrode in the liquid, but with the $3 \mathrm{D}$ design it is possible to adjust this. The total volume of the flow cell is equal to $110 \mu \mathrm{L}$. The thermal resistance, $\mathrm{R}_{\mathrm{th}}$, is then defined as $\left(\mathrm{T}_{1}-\mathrm{T}_{2}\right) / \mathrm{P}$. P is calculated is as the voltage squared over the internal resistance $(22 \mathrm{Ohm})$. The voltage is monitored by the thermocouple device, temperature $\mathrm{T}_{1}$ was kept constant at $37.00^{\circ} \mathrm{C}$.

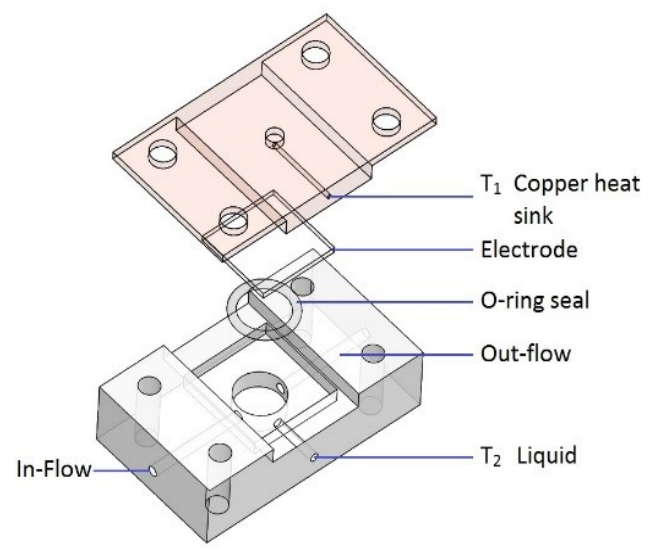

Figure 1. Technical drawing of the flow cell, demonstrating the in -and outlet, position of the thermocouples $\mathrm{T}_{1}$ and $\mathrm{T}_{2}$, O-ring used to seal off the sample and the copper block on top that acts as a heat sink. With eight connections to the thermocouple device, there is the possibility to develop an assay format.

\section{RESULTS AND DISCUSSION}

Binding affinity was determined by mixing polymer particles with solutions (phosphate buffered saline solutions of $\mathrm{pH}=7$ ) of known neurotransmitter concentrations. After filtering off the MIP and NIP powders, the absorbance in the solution was determined and used to calculate how much had bound to the particles. To quantify the specificity of the MIP towards it target, the imprint factor (IF) is used which is defined as the amount bound to the MIP divided over the amount bound to the NIP at a certain concentration.

For the noradrenaline MIP, at $\mathrm{pH}=7$ the solutions were unstable and the neurotransmitter oxidized. At $\mathrm{pH}=6$, better results were obtained with a max binding of $25 \mu \mathrm{mol} / \mathrm{g}$ and an imprint factor of approximately 1.6.

The optimized MIP mixture for caffeine detection, compromised of a 1:1 ratio of acrylamide and methacrylic acid, exhibited better specificity towards it target. A IF of 2.5 was achieved at $\mathrm{pH}=7$, with a maximum binding capacity of $50 \mu \mathrm{mol} / \mathrm{g}$. This is potentially because a combination of monomers is better able to bind targets if they have different hydrogen bond donor and acceptor groups on the molecule. 
The results for dopamine are summarized in Table 2. To test the selectivity, the response of the MIP towards caffeine and noradrenaline was also measured and only minimal binding ( $\max 15 \mu \mathrm{mol} / \mathrm{g}$ ) was observed.

Table 2. Binding capacity and specificity of MIPs developed for dopamine

\begin{tabular}{|c|c|c|}
\hline & $\begin{array}{c}\text { Max binding capacity } \\
(\mu \mathrm{mol} / \mathrm{g})\end{array}$ & $\begin{array}{c}\text { Imprint Factor (IF) } \\
-\end{array}$ \\
\hline MIP 1 & 49.0 & 1.1 \\
\hline MIP 2 & 60.8 & 1.6 \\
\hline MIP 3 & 110.0 & 1.9 \\
\hline MIP 4 & 41.0 & 1.1 \\
\hline MIP 5 & 58.0 & 1.5 \\
\hline MIP 6 & 95.0 & 1.9 \\
\hline
\end{tabular}

The polymers produced with solely HEMA had the lowest amount of binding compared to the other monomer compositions. A potential explanation is that HEMA offers only one charged group for interaction, while itaconic acid has a higher charge density to increase interactions between monomer and template. The combination of itaconic acid and HEMA yielded in the highest binding and IF. The porogen did not seem to have a significant effect on template binding.

The produced MIP-SPEs were produced with a 30\% mass of particulate vs ink ratio. MIPs were visible (Scanning Electron Microscopy) on the surface of the SPE but it was difficult to quantify since there was little contrast between the graphite based ink and the polymer. In the future, it might be worth considering adding a (fluorescent) label onto monomer or initiator to provide accurate values of the surface coverage. After stabilizing in a buffered solution, solutions with increasing concentration of neurotransmitter were added. The temperature in the set-up was kept at $37.00^{\circ} \mathrm{C}$ to mimic body conditions. After addition of a solution that was kept at room temperature, a spike in the thermal resistance is observed after which the signal gradually goes down. This corresponds to the feedback loop of the set up that will adjust the temperature back to its original value. For the NIP-SPEs, after stabilization the signal will then return to its original baseline value. For the MIP-SPEs, gradual increases in the thermal resistance are observed which is due binding of the template in the porous structure of the MIP that blocks heat-transfer through the surface (described in pore-blocking model, ref 6). A typical example of a heat-transfer measurement is shown in Figure 2. Dose-response curves are constructed to determine the limit of detection. For noradrenaline, it was not possible to determine this due to instabilities of the signal in phosphate buffers. For the caffeine MIP, it was estimated to be around $\sim 50 \mathrm{nM}$, while for the dopamine MIP this was around $25 \mathrm{nM}$ in buffered solution. Limit of detection depends on affinity of the MIP towards its target, but also towards how compatible the polymers are with the ink that is used in the screenprinting process. The limit of detection was at least an order of magnitude better compared to when electrochemical (Cyclic Voltammetry) measurements were performed on the functionalized SPEs. 


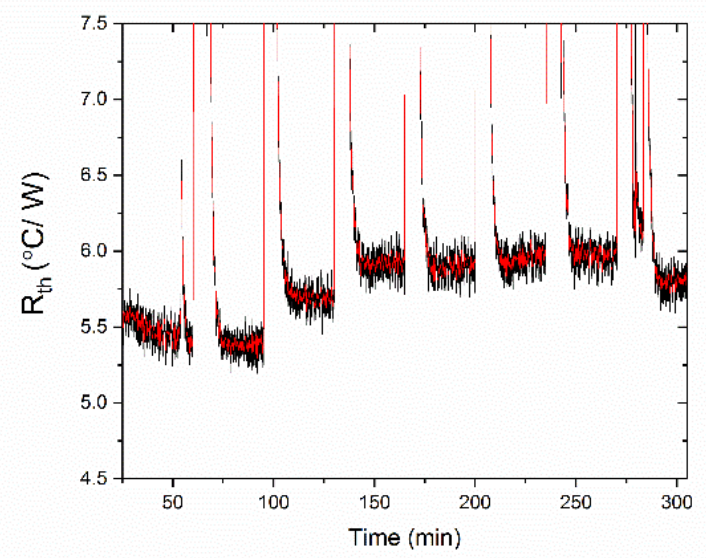

Figure 2. Thermal resistance for the MIP-SPEs printed for caffeine in time. The sensor was stabilized in PBS for 50 min, followed by additions of the following solutions with concentrations $0.05,0.1,0.15,0.25,0.5,0.75 \mathrm{mM}$. The red line shows a percentile filter (average 10 points), which reduces LoD to $\sim 25 \mathrm{nM}$.

\section{CONCLUSIONS}

MIPs for the neurotransmitters noradrenaline, caffeine and dopamine were developed. These were integrated into SPEs by direct mixing of the polymer particles with the ink, which is a fast and scalable production strategy. It was possible to determine neurotransmitter levels in the nanomolar regime using thermal detection, which is promising fast and low-cost sensing strategy with high commercial potential.

\section{ACKNOWLEDGMENTS}

WE WOULD LIKE TO THANK THE ENGINEERING AND PHYSICAL SCIENCES RESEARCH COUNCIL (EP/N001877/1) FOR FUNDING.

\section{REFERENCES}

111 K. Hawnt and K. Moshach. Chem. Rev. 100, 2495 (2000).

[2.1 G. Wulff. Chem. Rev. 102. 1. (200) 2).

131 D. A. Snivak. Adv. Drus. Del. Rev. 57. 1779. (2.005).

[4] S. Casadin. I.W. I.nwdon. K. Retlem. I.T. Ueta. C.W. Foster. T.I Cleii. R. van Grinsven, O.B. Si1tcliffe. C.F. Ranks and M. Peeters. Chem. F.no. I. 315. 459. (2017)

[5] B. van Grinsven. K Fersels. M Peeters. P I.nsada-Perez. T. Vandenryt, T.J. Cleij and P. Wagner, ACS Annl. Mater. Interfaces. 6. 16. 13309. (2014).

[6] L. Ye, P.A.G. Cormack and K. Mosbach, Anal. Commun, 36, 35, (1999). 